\title{
Relationship between tumor viability during treatment and the clinical outcomes of patients with bladder/prostate rhabdomyosarcoma: a single-center experience
}

\author{
Yun-peng $\mathrm{Li}^{1 \oplus}$, Hong-cheng Song ${ }^{1 \oplus}$, Xiao-li $\mathrm{Ma}^{2 \oplus}$, Le-jian $\mathrm{He}^{3 \oplus}$, Wei-ping Zhang ${ }^{1 \odot}$ \\ Departments of ${ }^{1}$ Urology, ${ }^{2}$ Hematology Oncology Center and ${ }^{3}$ Pathology, Beijing Children's Hospital, Capital Medical University, \\ National Center for Children's Health, Beijing, China.
}

\begin{abstract}
Background. To analyze the relationship between tumor viability in specimens retrieved at second-look procedures (SLPs) and clinical outcomes in patients with bladder/prostate rhabdomyosarcoma (BP RMS).

Methods. We retrospectively analyzed patients' characteristics, times between diagnoses and SLPs, types of SLPs, the pathological findings, and clinical outcomes between January 2003 and May 2014.

Results. A total of 29 patients underwent at least one SLP before completing chemotherapy, including 24 boys and 5 girls. The mean age was 36 months. No patients with clinical/radiographic complete responses (CRs) had viable tumor cells and 7/18 patients (38.9\%) without CR had no viable tumor cells. Seven patients experienced tumor relapse, progression, and metastasis, and three of these survived. Five-year event-free survival (EFS) rates were $88.5 \%$ in 18 patients without viable tumor at SLPs and 54.5\% in 11 patients with viable tumor (Cox proportional hazards adjusted $\mathrm{P}=0.045)$. The respective five-year overall survival (OS) rates were $94.1 \%$ and 72.7\% (Cox proportional hazards adjusted $\mathrm{P}=0.175$ ).

Conclusions. EFS was increased in patients with BP RMS having no viable tumor cells; however, OS was comparable in patients with and without viable tumor cells. Patients who achieved CR during the treatment generally had no viable tumor cells.
\end{abstract}

Key words: bladder/prostate, outcome, rhabdomyosarcoma, second-look procedures.

Rhabdomyosarcoma (RMS) is the most common soft tissue sarcoma in children, with an incidence of 4.4-6/1000000 per year. ${ }^{1,2}$ The prognosis of patients with bladder/prostate rhabdomyosarcoma (BP RMS) has improved in recent decades, and the overall survival rate for localized disease is approximately $84 \% .^{3}$

During treatment, the response to previous therapy may vary in patients with RMS. Some patients achieved CR while others had residual tumor mass on imaging. ${ }^{4,5}$ Second-look procedures (SLPs) are recommended to evaluate

\footnotetext{
$\triangle$ Wei-ping Zhang

zwp59616406@163.com
}

Received 31st May 2020, revised 17th July 2020, accepted 25th August 2020. the response to previous therapy, to control the progression of disease or to eliminate residual tumor tissue..$^{6-8}$ Some reports found viable tumor cells in specimens retrieved at SLPs, ${ }^{9}$ causing confusion as to whether this affected survival, and sometimes this led to radical surgery. ${ }^{10}$ We hypothesized that the presence of viable tumor cells would indicate that the tumor responded poorly to previous therapy, thereby having an adverse effect on treatment outcome.

\section{Material and Methods}

We retrospectively analyzed the medical records of patients with BP RMS treated in our institution between January 2003 and May 2014. Patients who underwent SLPs were included and those diagnosed with metastatic disease 
prior to treatment were excluded. Demographic data, types of SLPs, tumor viability and survival status were documented. The study was approved by the Medical Ethics Committee of our center (No. 2020-Z-016).

Our treatment protocol was derived from the Third Intergroup Rhabdomyosarcoma study (IRS-III). ${ }^{11}$ The chemotherapy regimens included vincristine, actinomycin $\mathrm{D}$, and cyclophosphamide (VAC). According to the treatment protocol, we performed SLPs at around 20 weeks to confirm the response to previous therapy, so as to facilitate subsequent treatment based on pathological findings. SLPs were defined as any procedures conducted after the initial biopsy, and open procedures were advised. Radiotherapy (RT) was administered based on the tumor response to prior chemotherapy and the pathological findings at SLPs. Tumor responses were categorized as complete response $(\mathrm{CR}$, no residual mass detected on radiographic films or during the SLPs), partial response (PR, a more than 50\% decrease in tumor mass), no response (NR, a decrease of $<50 \%$ and an increase of $<25 \%$ of tumor tissues), and progressive disease (PD, an increment of more than $25 \%$ in tumor mass). Only cells consistent with RMS on histology were identified as viable tumor cells. ${ }^{12}$ Mature rhabdomyoblastic cells were not considered evidence of viable tumor cells. ${ }^{13}$ The final pathological results were confirmed by two expert pathologists.

In situations where patients underwent more than one procedure after the initial biopsy, tumor viability at the first surgery was documented, because we believed this was a relatively accurate reflection of previous therapy and it might influence the subsequent treatment plan.

Overall survival (OS) was defined as the time from diagnosis to death from any cause. Eventfree survival (EFS) was defined as the time from diagnosis to relapse, progression, metastasis or death. There were no significant differences in OS or EFS using the time at SLPs as the initial time of follow-up when compared with values using the time at diagnosis. Therefore, the time at which SLPs were performed was used as the initial value for comparing the EFS and OS distributions, rather than the time at diagnosis, in order to avoid potential bias when comparing outcomes based on patient and disease characteristics. ${ }^{6}$ Kaplan-Meier curves were used to calculate the distributions of OS and EFS. Discrepancies between survival curves, based on whether viable tumor cells were present and whether the patients achieved $\mathrm{CR}$, were analyzed using the log-rank test. The chi-square test was used to compare the distributions of categorical characteristics. Previous studies found tumor size and invasive tumors were independent predictors for EFS and OS. ${ }^{3}$ To avoid other confounding factors, we used a Cox proportional hazards regression model, which incorporated age, tumor size, and invasiveness, to assess the differences of EFS and OS between different groups, if appropriate. Statistical analysis was conducted using SPSS 25.0 statistics software. Differences were considered statistically significant when $\mathrm{p}$ $<0.05$.

\section{Results}

Fifty patients were treated in our center during the study period, and 29 (58\%) met the inclusion criterion, including 24 boys and five girls. The mean age at diagnosis was 36 months \pm 33 months. Patient characteristics are illustrated in Table I. All patients were diagnosed with gross residual masses, and localized embryonal BP RMS, which were classified as IRS-III RMS based on the surgical-pathologic group system. The median time between diagnosis and SLPs was 5 months (range: 4-7 months), and the median time of follow-up was 7.5 years (range: 0.9-14.3 years).

Biopsy was performed in 15 patients, tumor resection in eight patients, partial cystectomy in one patient, and cystectomy in five patients. Eleven patients achieved CR on imaging before SLPs. None had viable tumor cells and none 
relapsed subsequently. Three patients received RT before SLPs and the remainder had never received RT. Seven out of 18 (38.9\%) patients without $\mathrm{CR}$ on imaging had no viable tumor cells (Table II). Two patients received RT before SLPs and two received RT subsequently. Among the remaining patients with viable tumor cells, three received RT before SLPs and four underwent RT subsequently. All patients continued chemotherapy after SLPs. The proportion of patients with no viable tumor cells was $62.1 \%(18 / 29)$. Among the patients with viable tumor cells, cells with rhabdomyoblastic differentiation were observed in the specimens retrieved from three patients. However, mature rhabdomyoblasts were observed in none of the patients without viable tumor cells. There

Table I. Characteristics of patients who underwent SLPs during treatment.

\begin{tabular}{lc}
\hline Variables & Number \\
\hline Age & 4 \\
$\quad<1$ year & 25 \\
$>1$ year & \\
Gender & 24 \\
$\quad$ Male & 5 \\
Female & \\
Tumor size & 20 \\
$\quad<5$ cm & 9 \\
$>5 \mathrm{~cm}$ & \\
Types of SLPs & 15 \\
Biopsy & 8 \\
Tumor resection & 1 \\
Partial cystectomy & 5 \\
Radical surgery & \\
\hline
\end{tabular}

SLPs $=$ second-look procedures.

Table II. Tumor viability, and tumor response to previous treatment.

\begin{tabular}{lcc}
\hline Tumor Response & No Viable Tumor & Viable Tumor \\
\hline CR & 11 & 0 \\
PR & 5 & 2 \\
NR & 2 & 8 \\
PD & 0 & 1
\end{tabular}

RT: radition therapy, CR: complete response, PR: partial response, NR: no response, PD: progression disease, SLPs: second-look procedures. was no significant difference in tumor viability between patients who received RT before SLPs and those who did not $(\mathrm{p}=1.000)$.

Tumor progression, relapse and/or distant metastasis occurred in two patients with PR, four patients with NR and one patient with PD after SLPs. Four of them received RT and five had viable tumor cells. Three patients experienced local recurrence after radical surgery and all of them had viable tumor cells. One patient died 6 months after the SLP and the other two patients survived event-free. One patient without viable tumor cells relapsed 5 months after completion of treatment, and was successfully treated with chemotherapy and RT. Two patients developed metastases, and both subsequently died including one patient without viable tumor cells. One patient with viable tumor cells died of disease progression. None of the patients with CR experienced any adverse events after SLPs.

The five-year EFS rates were $88.5 \%$ for patients without viable tumor cells and $54.5 \%$ for patients with viable tumor cells $(p=0.030)$ using the time at diagnosis as the beginning time of follow-up, while the five-year EFS rates were $88.5 \%$ for patients without viable tumor tissues and $54.5 \%$ for those with viable tumor tissues ( $p=0.031$ ) (Fig. 1) when using the time at SLPs as the beginning time of follow-up. After adjusting the data in terms of age, tumor size, and invasiveness, the EFS was prolonged significantly in patients without viable tumor cells ( $\mathrm{HR}=0.14$ for no viable tumor cells vs. viable tumor cells, $\mathrm{p}=0.045,95 \% \mathrm{CI}=0.02-0.96)$. The five-year OS rates were $94.1 \%$ and $72.7 \%$ among patients without viable tumor cells and those with viable cells, respectively $(\mathrm{p}=0.097)$, when using the time at diagnosis as the beginning time of follow-up. The five-year OS rates were $94.1 \%$ and $72.7 \%$ among patients without viable tumor cells and those with viable tumor cells, respectively ( $p=0.094$ ) (Fig. 2), when using the time at SLPs as the beginning time of followup. OS was also extended, but not significantly based on the comparisons adjusted for age, tumor size, and invasiveness ( $\mathrm{HR}=0.17$ for 


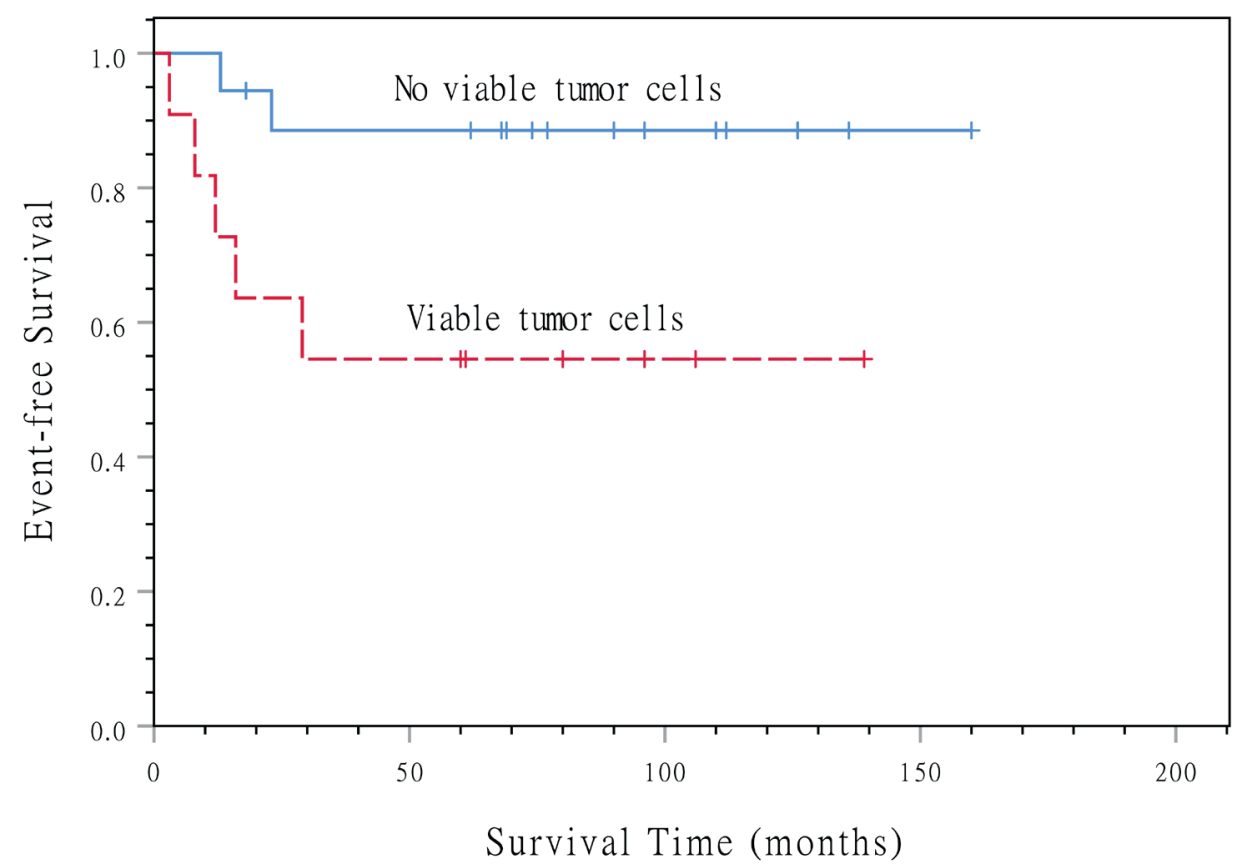

Fig. 1. Kaplan-Meier curve for EFS of patients who underwent SLPs during the treatment.

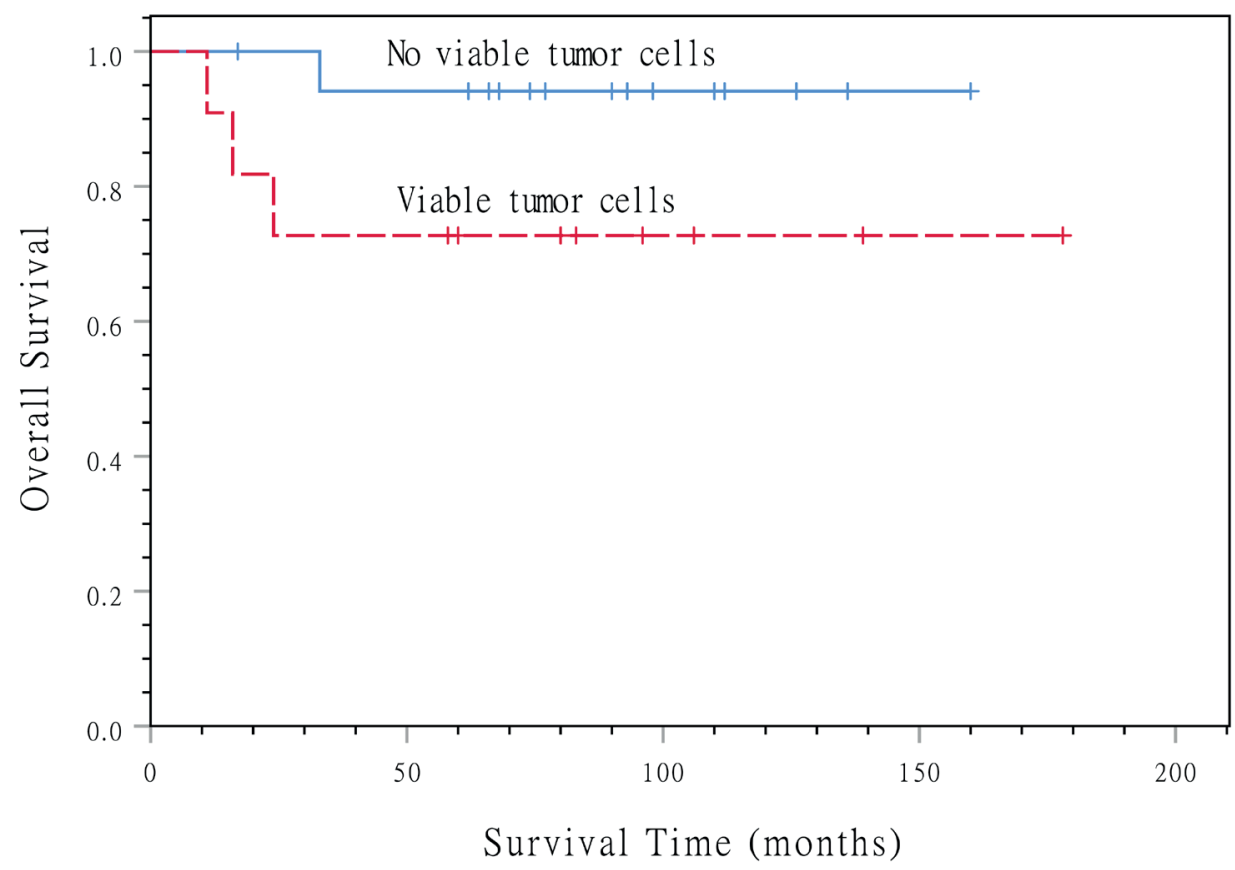

Fig. 2. Kaplan-Meier curve for OS of patients who underwent SLPs during the treatment.

no viable tumor cells vs. viable tumor cells, $\mathrm{p}=0.175,95 \% \mathrm{CI}=0.01-2.19)$. We also compared the differences of EFS and OS between patients with $C R$ and those without CR. The 5-year EFS rates were $100 \%$ in patients with $\mathrm{CR}$ and $61.1 \%$ in those without CR ( $p=0.025)$. The 5-year OS rates were $100 \%$ and $77.8 \%$, respectively $(p=$ $0.11)$. 


\section{Discussion}

Determining whether viable tumor cells existed in the residual masses and whether tumor viability would influence the clinical outcome appeared important for subsequent treatment. Our experience with BP RMS patients demonstrated that all the participants with $\mathrm{CR}$ were free from viable tumor cells and $38.9 \%$ of patients without CR had no viable tumor cells. The overall outcome was comparable between patients with viable tumor cells and those without viable tumor cells.

Our results were similar to those of previous reports. ${ }^{6}$ In the IRS-III study, SLPs were often performed around 20 weeks following chemotherapy and RT. ${ }^{11}$ Among the 109 patients who received SLPs in the IRS-III study, $88 \%$ of those with CR did not have viable tumor cells and the 3-year survival rate was $85 \%$. The respective percentage of patients without viable tumor cells was $75 \%$ in patients with PR and $28 \%$ in patients with less than PR; their 3-year survival rate was $83 \%$. Among the 14 patients who had viable tumor cells, the 3 -year survival rate was $62 \%{ }^{6}$ However, the authors did not assess the EFS nor compare the differences in OS. In the IRS-IV study, SLPs were recommended to be undergone at 46 or 47 weeks in patients still having tumor masses. ${ }^{6} \mathrm{~A}$ total of 13/14 (93\%) patients with CR presented no viable tumor cells and 24/59 (41\%) patients without $C R$ had no viable tumor cells in the IRSIV study. ${ }^{6}$ Both our study and the IRS-IV study showed patients without viable tumor cells had increased EFS, not OS; we further demonstrated that the tumor viability did not influence the outcome of patients with BP RMS.

Whether the response to the previous treatment modality affects the outcome and whether it influences further treatment plans are becoming important concerns. In a report from the Children's Oncology Group (COG), the authors discovered that, among the 338 patients with Group III RMS, the 5-year FFS was similar between patients with $C R, P R$, and
NR. ${ }^{5}$ Another report from the International Society of Pediatric Oncology (SIOP) MMT-95 also reported no significant differences in FFS and OS between different response groups. ${ }^{4}$ Therefore, the authors proposed that treatment adaptations based on early response should not be incorporated into future studies. ${ }^{4}$ However, patients with BP RMS in our study who achieved $\mathrm{CR}$ had longer EFS, than those without CR. These inconsistencies can be explained by the fact that our study's sample size was relatively small and that confounding factors were not controlled for. The results agreed with our pathological findings at SLPs, which found EFS was significantly prolonged in patients without viable tumor cells.

The disappearance of tumor masses on imaging or viable tumor cells on the specimens are not always reliable factors to predict any potential relapse. In a study from SIOP MMT- $84,{ }^{7}$ the authors discovered $51 \%(27 / 52)$ of patients with RMS developed localized recurrence despite biological confirmation of complete remission during the treatment, in contrast to $48 \%(19 / 39)$ in non-biopsied patients. ${ }^{7}$ Nonetheless, the recurrence rate was $14 \%(1 / 7)$ in patients with BP RMS. ${ }^{7}$ The low recurrence rate in BP RMS patients with $\mathrm{CR}$ on pathologic screening was confirmed in our study, suggesting that none of the patients who attained CR without viable tumor cells relapsed, while another study found that only $22 \%$ (2/9) of patients with BP RMS who achieved CR on pathologic screening experienced tumor relapse. ${ }^{6}$ It appears that the recurrence rates in different sites might vary for patients with $\mathrm{CR}$ on pathologic finding. Hays et al. ${ }^{9}$ found that seven of 18 patients without viable tumor cells at second surgeries experienced tumor relapse; the authors argued that negative pathological findings did not predict any subsequent recurrence, given that previous therapy made it difficult to identify viable tumor cells.

RT seemed to have a pivotal role in local control of the disease. Cecchetto et $\mathrm{al}^{8}{ }^{8}$ examined the relationship between radiation 
and tumor relapse in patients who underwent microscopically complete second look operations of gross residual masses and found that two of 12 patients with RT relapsed; by contrast, relapse occurred in 11 out of 27 patients without RT and five-year progressionfree survival rates were $83 \%$ and $58 \%$ in patients with RT and those without RT, respectively (No $\mathrm{p}$ value was provided). ${ }^{8}$ Raney et $a .^{6}$ reported that $32 \%(13 / 41)$ of patients with RT administered before SLPs had viable tumor cells, compared to $78 \%(14 / 18)$ in patients without RT $(p=0.001)$. In our study, we found there was no significant difference in tumor viability at SLPs between patients who received $\mathrm{RT}$ and those who did not. The variation might be explained by the fact that the results of these studies came from different timing. Unlike the study carried out by Raney et al. ${ }^{6}$, whose results were obtained from final procedures, we used pathological findings at initial SLPs. Despite these negative findings, we continue to hold the opinion that RT permits better local control, and nearly half of the patients received RT in our study.

It is worth mentioning that there are some limitations in our study. This study was a retrospective review with a small number of patients. There were several factors that could possibly affect the prognosis. That said, the adjusted model only incorporated patients' age, tumor size, and invasiveness due to the small number of patients; therefore, there might be some other variables such as lymph node involvement and the status of radiotherapy that could have influenced the outcomes. The timing and reasons for SLPs may vary from one patient to another. Nevertheless, our study shed light on the effect of tumor viability on the survival of patients with BP RMS. Further functional imaging studies such as F-18 fluorodeoxyglucose positron emission tomography (FDG-PET) are required to investigate the biological activity of residual masses. In this way, selected patients may be spared additional surgery. ${ }^{14}$
Among patients with BP RMS, the five-year EFS was increased in patients without viable tumor cells. OS was also increased, but not significantly. Disappearance of tumor masses was always associated with the absence of viable tumor cells at SLPs. Thus, avoiding an additional SLP to confirm the results in patients with CR appears reasonable. Notably, $38.9 \%$ of patients with residual masses had no viable tumor cells at SLPs. Further studies are necessary to verify these results.

\section{Acknowledgement}

The authors would like to thank Dr. Libing $\mathrm{Fu}$ and Dr. Nan Zhang from the Department of Pathology of our center for reviewing the specimens.

\section{Author contribution}

The authors confirm contribution to the paper as follows: study conception and design: Hongcheng Song, Wei-ping Zhang; data collection: Yun-peng Li, Le-jian $\mathrm{He}$; analysis and interpretation of results: Xiao-li Ma, Le-jian He, Wei-ping Zhang; draft manuscript preparation: Yun-peng Li, Hong-cheng Song. All authors reviewed the results and approved the final version of the manuscript.

\section{Ethical approval}

The study was approved by the Medical Ethics Committee of our center (No. 2020-Z-016).

\section{Sources of financial}

None.

\section{Conflicts of interest}

The authors declare no conflict of interest. 


\section{REFERENCES}

1. Perez EA, Kassira N, Cheung MC, Koniaris LG, Neville HL, Sola JE.Rhabdomyosarcoma in children: a SEER population based study. J Surg Res 2011;170: e243-e251.

2. Dasgupta R, Fuchs J, Rodeberg D. Rhabdomyosarcoma. Semin Pediatr Surg 2016; 25: 276-283.

3. Rodeberg DA, Anderson JR, Arndt CA, et al. Comparison of outcomes based on treatment algorithms for rhabdomyosarcoma of the bladder/ prostate: combined results from the Children's Oncology Group, German Cooperative Soft Tissue Sarcoma Study, Italian Cooperative Group, and International Society of Pediatric Oncology Malignant Mesenchymal Tumors Committee. Int J Cancer 2011; 128: 1232-1239.

4. Vaarwerk B, van der Lee JH, Breunis WB, et al. Prognostic relevance of early radiologic response to induction chemotherapy in pediatric rhabdomyosarcoma: a report from the International Society of Pediatric Oncology Malignant Mesenchymal Tumor 95 study. Cancer 2018; 124: 1016-1024.

5. Rosenberg AR, Anderson JR, Lyden E, et al. Early response as assessed by anatomic imaging does not predict failure-free survival among patients with Group III rhabdomyosarcoma: a report from the Children's Oncology Group. Eur J Cancer 2014; 50: 816-823.

6. Raney B, Stoner J, Anderson J, et al; Soft-Tissue Sarcoma Committee of the Children's Oncology Group. Impact of tumor viability at second-look procedures performed before completing treatment on the Intergroup Rhabdomyosarcoma Study Group protocol IRS-IV, 1991-1997: a report from the children's oncology group. J Pediatr Surg 2010; 45: 2160-2168.
7. Godzinski J, Flamant F, Rey A, Praquin MT, Martelli $\mathrm{H}$. Value of postchemotherapy bioptical verification of complete clinical remission in previously incompletely resected (stage I and II pT3) malignant mesenchymal tumors in children: International Society of Pediatric Oncology 1984 Malignant Mesenchymal Tumors Study. Med Pediatr Oncol 1994; 22: 22-26.

8. Cecchetto G, Carretto E, Bisogno G, et al. Complete second look operation and radiotherapy in locally advanced non-alveolar rhabdomyosarcoma in children: a report from the AIEOP soft tissue sarcoma committee. Pediatr Blood Cancer 2008; 51: 593-597.

9. Hays DM, Raney RB, Crist WM, et al. Secondary surgical procedures to evaluate primary tumor status in patients with chemotherapy-responsive stage III and IV sarcomas: a report from the Intergroup Rhabdomyosarcoma Study. J Pediatr Surg 1990; 25: 1100-1105.

10. Stein R, Frees S, Schroder A, et al. Radical surgery and different types of urinary diversion in patients with rhabdomyosarcoma of bladder or prostate--a single institution experience. J Pediatr Urol 2013; 9(6 Pt A): 932-939.

11. Crist W, Gehan EA, Ragab AH, et al. The Third Intergroup Rhabdomyosarcoma Study. J Clin Oncol 1995; 13: 610-630.

12. Parham DM, Barr FG.Classification of rhabdomyosarcoma and its molecular basis. Adv Anat Pathol 2013; 20: 387-397.

13. Arndt CA, Hammond S, Rodeberg D, Qualman S. Significance of persistent mature rhabdomyoblasts in bladder/prostate rhabdomyosarcoma: results from IRS IV. J Pediatr Hematol Oncol 2006; 28: 563567.

14. Wu HY.The surgical management of paediatric bladder and prostate rhabdomyosarcoma. Arab J Urol 2013; 11: 40-46. 